\title{
Wide-Range Phase Control with Constant Attenuation by Adjustable Impedance in a Resistance-Loaded Bridged- Tee Network
}

\author{
By Myron G. Pawley
}

\begin{abstract}
Phase may be shifted through a wide range without change in attenuation by means of a properly designed bridged-tee network. Equations are presented, and the necessary relations between circuit constants are deduced for phase control using an adjustable resistance, inductance, or capacitance located in a suitably selected branch of a bridged-tee network.
\end{abstract}

\section{Introduction}

Bridged-tee circuits are widely used as null networks [1]. ${ }^{1}$ This paper shows that bridged-tee networks may be devised for phase shifting or phase modulating and gives the necessary relations between circuit constants when the phase displacement is produced by an adjustable resistance, inductance, or capacitance in the proper branch of the tee. Advantages of these circuits will be seen to include: wide-range phase shift without change in attenuation; common ground for input and output circuits, as well as for one end of a single adjustable control impedance; large change in phase with small variation in control reactance; ability to work into a low resistance load; stability; and simplicity.

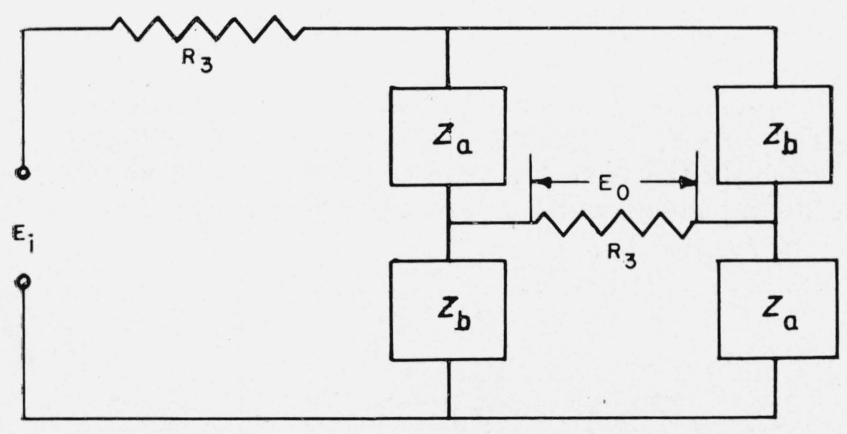

FiguRE 1. Symmetrical lattice network with resistive load.

A good general approach to the problem is to first consider the lattice network shown in figure 1 . It can be shown that the transfer function [2] for this circuit can be written [3] as

$$
\frac{E_{o}}{E_{i}}=\frac{R_{3}}{2}\left(y_{a o}-y_{b o}\right)
$$

where

$$
\left.\begin{array}{l}
y_{a o}=\frac{1}{Z_{a o}} ; \quad y_{b o}=\frac{1}{Z_{b o}} ; \\
Z_{a o}=R_{3}+Z_{a} ; \quad Z_{b o}=R_{3}+Z_{b}
\end{array}\right\}
$$

${ }^{1}$ Figures in brackets indicate the literature references at the end of this paper.
For constant attenuation, $\alpha$, with phase shift produced by variation in resistance or reactance in the circuit, one may write

$$
\frac{E_{0}}{E_{i}}=\alpha \epsilon^{j \beta},
$$

where $\alpha$ is a constant independent of the variable that changes the phase angle $\beta$. Hence, one may represent this transfer function geometrically as a circular locus with pole at the center.

\section{Variable Reactance Control of Phase with Constant Attenuation}

If we desire to use an adjustable reactance as the control element, we can examine the expressions in terms of the components that $Z_{a}$ and $Z_{b}$ of figure 1 require to preserve the form of eq 3 .

Referring to figure 1 and to eq 2 , if

and

$$
\left.\begin{array}{l}
Z_{a}=R_{a} \\
Z_{b}=R_{b}+j X
\end{array}\right\}
$$

where $X$ may be inductive or capacitative, we obtain the lattice network of figure 2, a. Here,

$$
y_{a 0}=\frac{1}{R_{3}+R_{a}}
$$

and

$$
\left.\begin{array}{rl}
y_{b o} & =\frac{1}{\left(R_{3}+R_{b}\right)} \sqrt{1+\left(\frac{X}{R_{3}+R_{b}}\right)^{2}} \epsilon^{-i \operatorname{arc} \tan \frac{X}{R_{3}+R_{b}}} \\
& =\frac{1}{R_{3}+R_{b}} \cos \theta \cdot \epsilon^{-j \theta}
\end{array}\right\}
$$

where

$$
\theta=\arctan \frac{X}{R_{3}+R_{b}} \cdot
$$




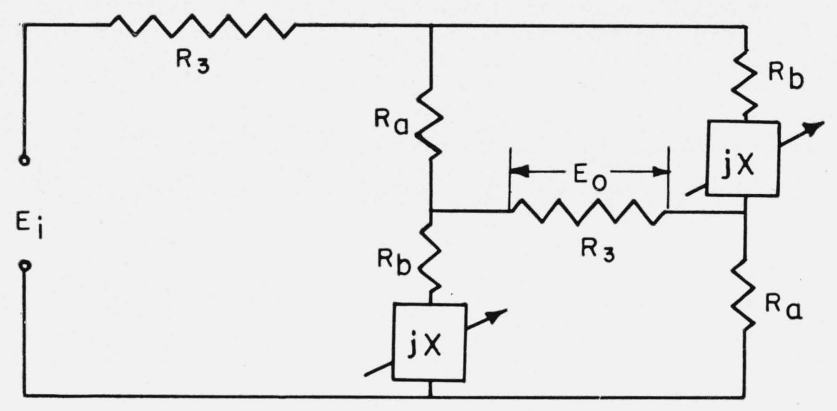

a

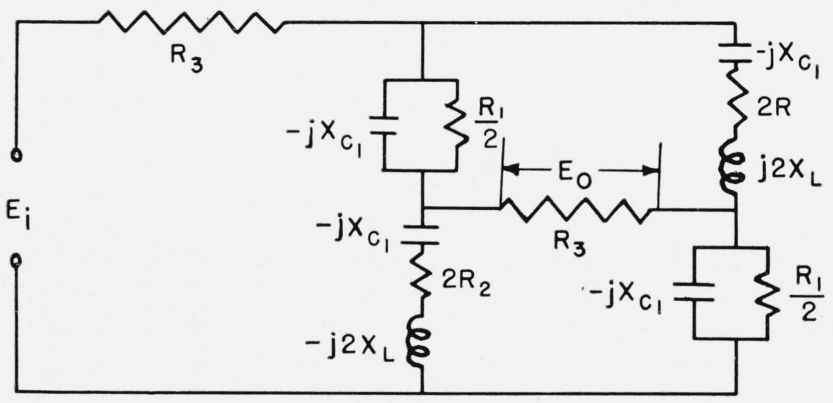

b

Figure 2. Lattice networks for variable reactance control of phase with constant attenuation.

A study of the geometry of the vectors representing the admittances $y_{a o}$ and $y_{b_{0}}$ shows that the desired phase sbift characteristics will be obtained in the circuit of figure 2, a, provided

$$
R_{a}=R_{3}+2 R_{b} .
$$

In this case,

$$
\begin{aligned}
y_{a o}-y_{b o} & =\frac{1}{R_{3}+R_{b}}\left[\frac{1}{2}-\cos \theta(\cos \theta-j \sin \theta)\right] \\
& =-\frac{1}{2\left(R_{3}+R_{b}\right)}[\cos 2 \theta-j \sin 2 \theta] \\
& =-\frac{1}{2\left(R_{3}+R_{b}\right)} \epsilon^{-j 2 \theta},
\end{aligned}
$$

and eq 1, which represents the transfer function for the circuit of figure 1 , and for that of figure 2 , a, may be written

$$
\left.\begin{array}{l}
\frac{E_{o}}{E_{i}}=-\frac{R_{3}}{4\left(R_{3}+R_{b}\right)} \epsilon^{-j 2 \theta}, \\
\text { where } \\
\theta=\arctan \frac{X}{R_{3}+R_{b}} \cdot
\end{array}\right\}
$$

Equation 7 is now in the form of eq 3, and therefore represents the transfer function for a lattice network giving constant attenuation when the phase is shifted by variation in reactance $X$.

Hence, the circuit of figure 1 becomes the desired phase shift network, shown in figure 2, a, provided $Z_{a}$ and $Z_{b}$ are as given by eq 4 and provided the circuit constants are related as given in eq 6 .

The circuit shown in figure $2, \mathrm{~b}$, also will be shown to satisfy these conditions, and hence will represent an example with the desired characteristics. AIthough lattice networks cannot always be converted to equivalent bridged-tee networks, this particular circuit was chosen because it may be converted to an equivalent bridged-tee. The lattice network is not generally convenient as a phase shifter, with constant attenuation, because simultaneous variation of the reactances in two arms would be required. The equivalent bridged-tee has the advantage of requiring the variation of only one reactance element for shifting the phase and of having the ground common to one side of the input and output, as well as to one end of the variable control reactance.

Examination of figure 2, b, shows that if we make $X_{C_{1}} \gg R_{1}$,

$$
\left.\begin{array}{l}
Z_{a} \doteq \frac{R_{1}}{2}, \\
Z_{b}=2 R_{2}+j\left(2 X_{L}-X_{C_{1}}\right)
\end{array}\right\}
$$

These forms for $Z_{a}$ and $Z_{b}$ correspond to those given by eq 4 . If, in addition,

$$
\frac{R_{1}}{2}=R_{3}+4 R_{2},
$$

the condition given in eq 6 is satisfied, and the circuit of figure 2, b, provides an example of the desired phase shift network. From eq 4, 7, 8, and 9, the transfer function may now be written

where

$$
\left.\begin{array}{c}
\frac{E_{0}}{E_{i}}=-\frac{R_{3}}{4\left(R_{3}+2 R_{2}\right)} \epsilon^{-j 2 \theta}, \\
\theta=\arctan \frac{2 X_{L}-X_{C_{1}}}{R_{3}+2 R_{2}}
\end{array}\right\}
$$

Equation 10, therefore, represents the transfer function for the circuit of figure $2, b$, provided

and

$$
\left.\begin{array}{l}
X_{C_{1}} \gg R_{1}, \\
\frac{R_{1}}{2}=R_{3}+4 R_{2} .
\end{array}\right\}
$$

Under these conditions, simultaneous variation of the reactances in the circuit of the symmetrical lattice network of figure 2, b, shifts the phase without varying the attenuation.

An equivalent bridged-tee network [4] shown in figure 3 may be derived from the lattice of figure $2, b$. The variable shunt capacitor, shown connected with a dashed line, shows one means of varying the effec- 
tive reactance for producing phase shift. This will be described more fully later in this paper. The transfer function for this bridged-tee network (not including the added capacitative reactance $X_{C_{2}}$ ) is the same as that given in eq 10 for the corresponding lattice network of figure $2, \mathrm{~b}$.

In the bridged-tee circuit of figure 3 , the phase may be shifted by varying the inductance, for example, with an adjustable core reactor or with a saturable reactor. The attenuation will not remain constant unless the resistance $R_{2}$ of the reactor remains constant. In laboratory tests, $R_{2}$ was found not to change appreciably when the circuit reactance was changed by varying an external capacitance across the high-? inductor. With this mode of operation, the circuit performed closely to theoretical predictions.

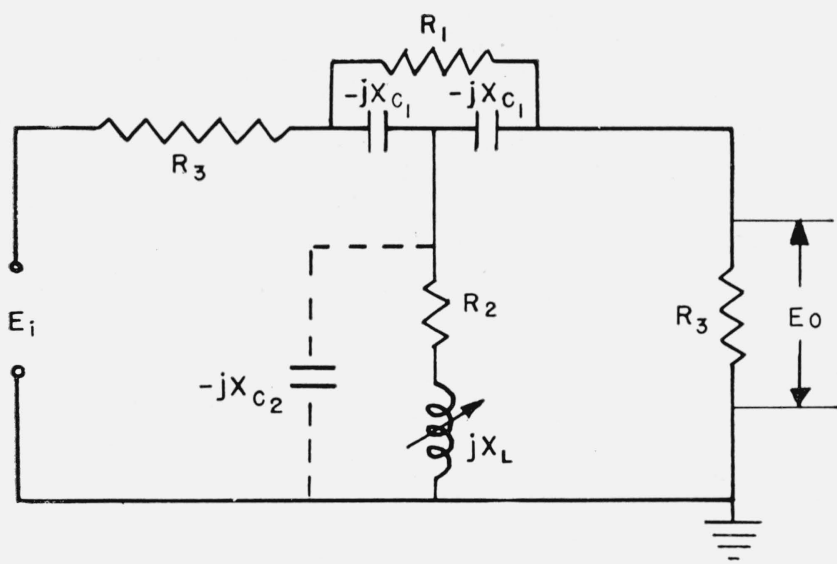

Figure 3. Bridged-tee equivalent to lattice network of figure $2, b$.

Examination of the transfer function, eq 10, for the bridged-tee of figure 3 shows that as the resistance $R_{2}$ of the inductor approaches zero, ( $Q$ approaches $\infty$ ) the attenuation approaches a minimum of one-fourth, and the phase sensitivity, with variation in reactance, approaches a maximum.

When $X_{L}$ is considered the variable in figure 3 , with $C_{2}=0$, it is convenient to write eq 10 in a different form:

Let

$$
\delta L=\frac{2 X_{L}}{X_{C_{1}}}=2 \omega^{2} C_{1} L
$$

In this case, eq 10 becomes

where

$$
\left.\begin{array}{c}
\frac{E_{o}}{E_{i}}=-\frac{R_{3}}{4\left(R_{3}+2 R_{2}\right)} \epsilon^{-j 2 \theta}, \\
\theta=\arctan \frac{\left(\delta_{L}-1\right) X C_{1}}{R_{3}+2 R_{2}} \cdot
\end{array}\right\}
$$

Equation 13, therefore, represents the transfer function for the bridged-tee of figure 3 , with $C_{2}=0$, and with $\delta_{L}$ proportional to $L$, as indicated by eq 12 . As explained previously, for constant attenuation with variable phase shift, the conditions given with eq 11 are necessary.

When $X_{C 2}$ is considered the variable in figure 3 , it is convenient to write eq 10 in another form:

Assume the $\mathrm{Q}$ of the inductor to be high, and that the network is adjusted for $X_{L}=X_{C_{1}} / 2$. Let

$$
\delta_{C}=\frac{X_{L}}{X_{C_{2}}}=\frac{X_{C_{1}}}{2 X_{C_{2}}}=\omega^{2} L C_{2} \ll 1 .
$$

If we replace $X_{L}$ in eq 10 by the parallel combination of $X_{L}$ and $X_{C 2}$ in figure 3 , then make $X_{L}=X_{C 1} / 2$, and substitute for $X_{C 2}$ from eq 14, eq 10 becomes

$$
\left.\begin{array}{c}
\frac{E_{o}}{E_{i}}=-\frac{R_{3}}{4\left(R_{3}+2 R_{2}\right)} \epsilon^{-j 2 \theta}, \\
\theta=\arctan \frac{X_{C_{1}} \delta_{C}}{R_{3}+2 R_{2}} \\
\delta_{C} \ll 1 .
\end{array}\right\}
$$

Equation 15 then represents the transfer function for the bridged-tee of figure 3 , with $\delta_{C}$ proportional to $C_{2}$, as indicated by eq 14 . As in the preceding case, for constant attentuation with variable phase shift, the conditions given with eq 11 are necessary.

Attenuation and phase characteristic curves, with phase shift and attenuation shown as functions of shunt capacitance $C_{2}$, are given in figure 4 , a, for the bridged-tee network of figure 4, c, operating at a frequency of $4,170 \mathrm{c} / \mathrm{s}$. Figure 5 , a, shows similar curves for the bridged-tee phase modulator of figure 5 , b, operating at $29.1 \mathrm{Mc} / \mathrm{s}$. These curves show the large changes in phase that may be secured by small changes in capacitance, particularly at the higher frequency. Figure 4, a, shows that, even at 4,170 $\mathrm{c} / \mathrm{s}, 90^{\circ}$ phase shift is produced by a shunt capacitance change of only $25 \mu \mu \mathrm{f}$. As shown in figure 5, a, at $29.1 \mathrm{Mc} / \mathrm{s}$, a phase shift of $90^{\circ}$ is produced by a capacitance change of only $2 \mu \mu \mathrm{f}$.

Inspection of eq 10, giving the transfer function for the networks of figures $2, \mathrm{~b}$, and 3 , shows that the attenuation is independent of frequency in the lattice network as well as in its equivalent bridged-tee network. Figure 4, b, shows the attenuation and phase curves for the bridged-tee of figure 4, c, when $C_{2}=0$ and the frequency is varied.

\section{Variable Resistance Control of Phase with Constant Attenuation}

If we desire to use a variable resistor as the control element for shifting phase, we can examine the components of $Z_{a}$ and $Z_{b}$ of figure 1 that will make eq. 1 assume the form of eq. 3 .

Referring to figure 1 and to eq. 2 , if

and

$$
\left.\begin{array}{l}
Z_{a}=j X_{a} \\
Z_{b}=R_{b}+j X_{b}
\end{array}\right\},
$$




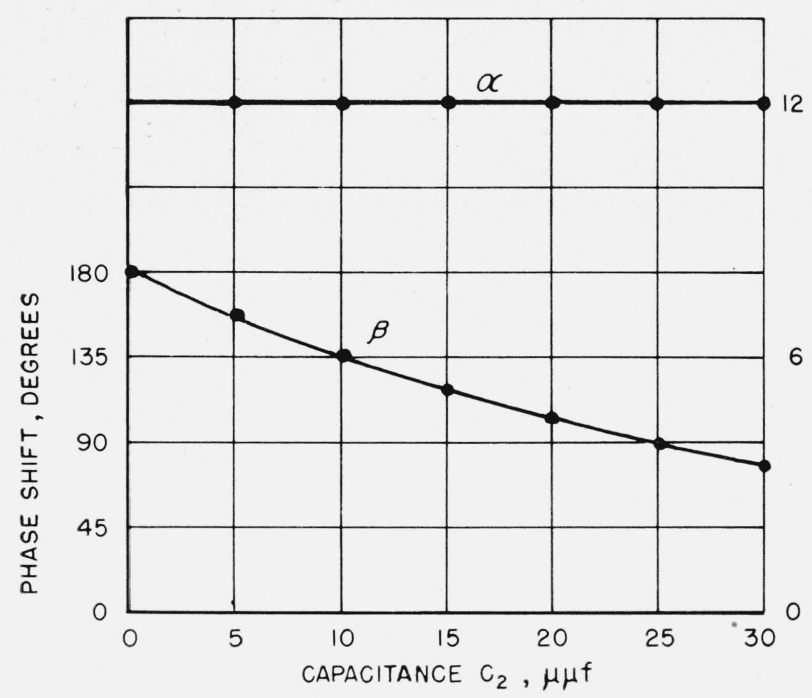

a

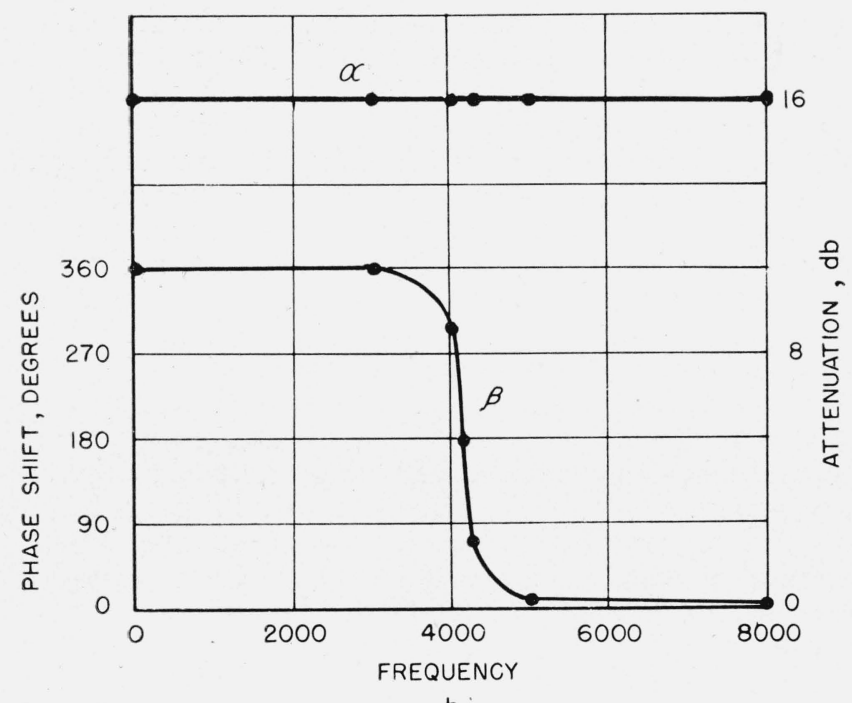

$5120 \Omega$

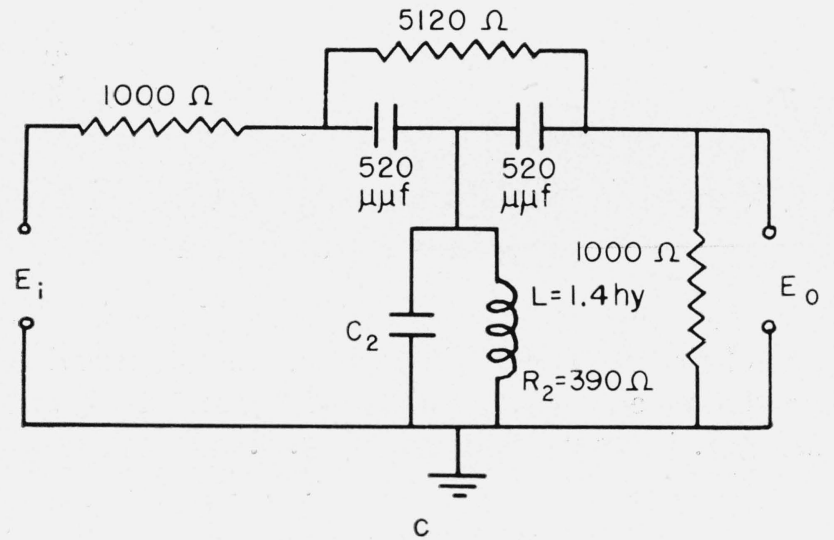

FIGURE 4. Attenuation and phase characteristics for bridged-tee network with $(a)$ variable reactance control $(f=4,170 \sim)$; (b) variable frequency $\left(\mathrm{C}_{2}=0\right)$.
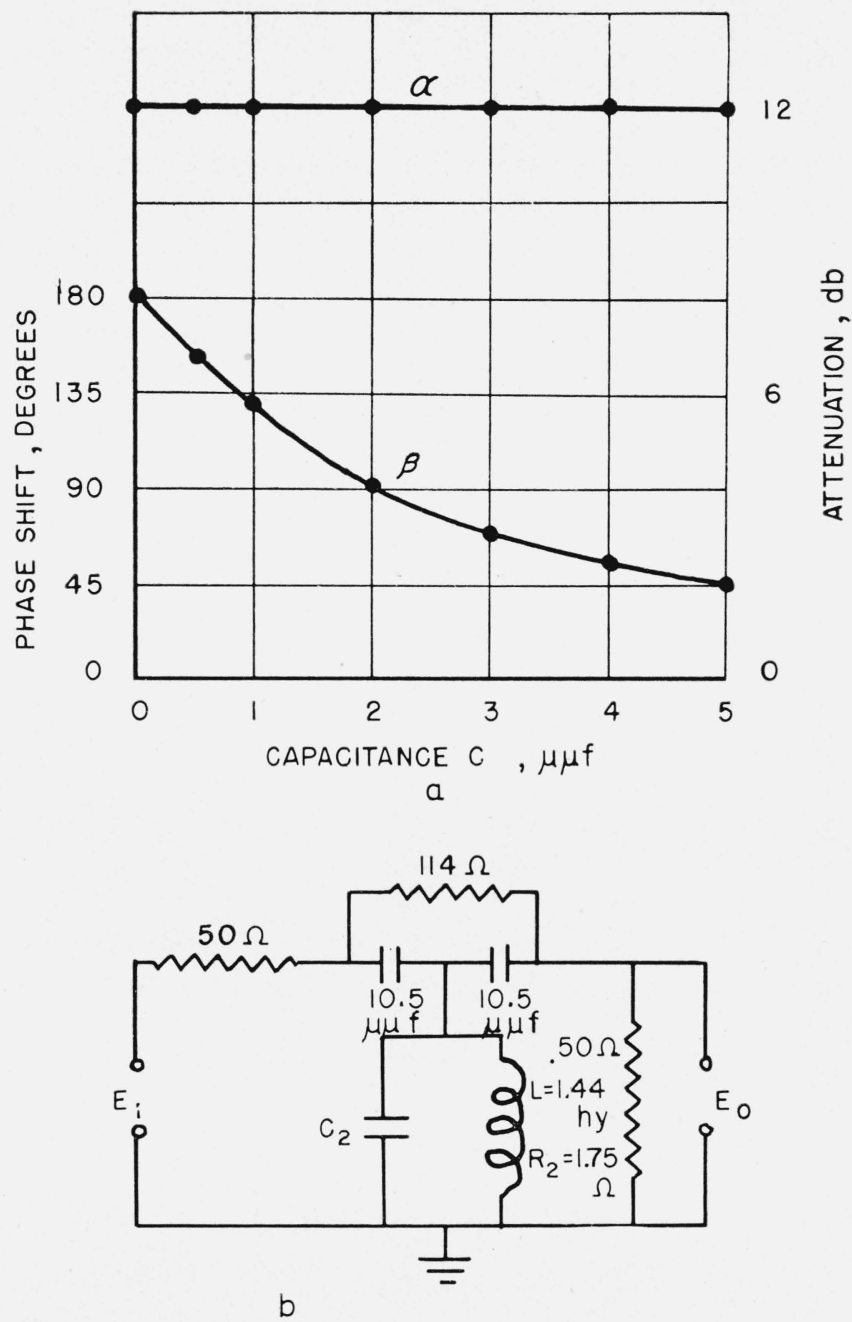

Figure 5. Attenuation and phase characteristics for bridged-tee network with variable reactance control $(f=29.1 \mathrm{Mc})$.

where $X_{a}$ and $X_{b}$ are of the same sign, we obtain the lattice network of figure 6 , a. If we assume $\left|X_{a}\right| \gg$ $R_{3}$,

and

$$
y_{a o}=-j \frac{1}{X_{a}}
$$

$$
\left.\begin{array}{rl}
y_{b o} & =\frac{1}{X_{b} \sqrt{\left(\frac{R_{3}+R_{b}}{X_{b}}\right)^{2}+1}} \epsilon^{-j \arctan \frac{X_{b}}{R_{3}+R_{b}}} \\
& =\frac{1}{X_{b}} \sin \theta \cdot \epsilon^{-j \theta},
\end{array}\right\}
$$

where

$$
\theta=\operatorname{arc} \tan X_{b} /\left(R_{3}+R_{b}\right) .
$$

A study of the geometry of the vectors representing the admittances $y_{a o}$ and $\mathrm{y}_{b o}$ shows that the desired 


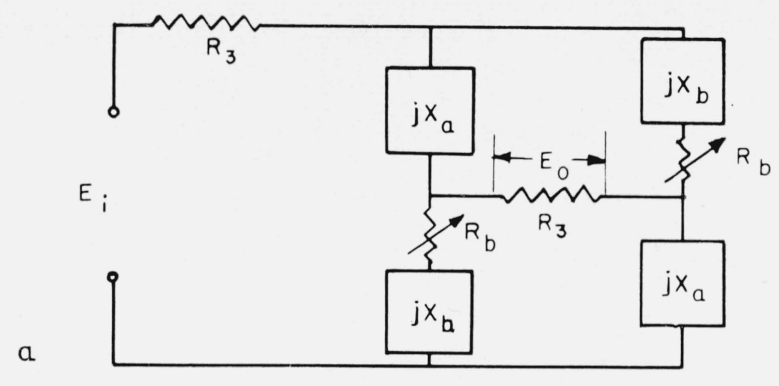

b

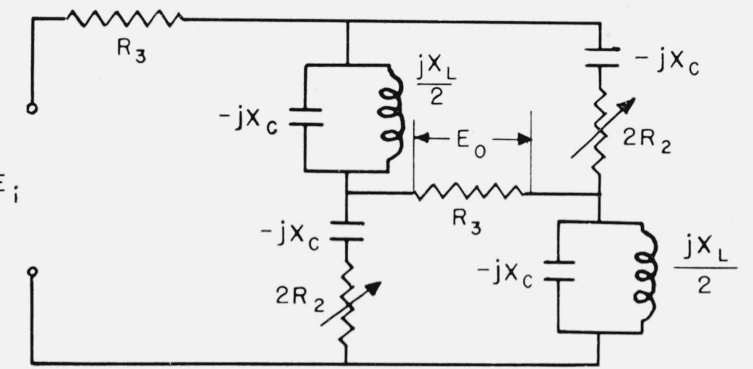

Figure 6. Lattice networks for variable resistance control of phase with constant attenuation. (a) $X_{a}$ and $X_{b}$ have same sign.

phase shift characteristics will be obtained in the circuit of figure 6 , a, provided

In this case,

$$
X_{a}=2 X_{b} .
$$

$$
\begin{aligned}
y_{a o}-y_{b o} & =\frac{1}{2 X_{b}}[-j-2 \sin \theta(\cos \theta-j \sin \theta)] \\
& =-\frac{j}{2 X_{b}}[\cos 2 \theta-j \sin 2 \theta] \\
& =-j \frac{1}{2 X_{b}} \epsilon^{-j 2 \theta},
\end{aligned}
$$

and eq. 1, which represents the transfer function for the circuit of figure 1, and for that of figure 6, a, may be written

where

$$
\left.\begin{array}{l}
\frac{E_{o}}{E_{i}}=-j \frac{R_{3}}{4 X_{b}} \epsilon^{-j 2 \theta}, \\
\theta=\arctan \frac{X_{b}}{R_{3}+R_{b}} .
\end{array}\right\}
$$

Equation 19 is now in the form of eq 3, and therefore represents the transfer function for a lattice network giving constant attenuation when the phase is shifted by adjusting the resistance $R_{b}$.

Hence, the circuit of figure 1 becomes the desired phase shift network, shown in figure $6 \mathrm{a}$, provided the necessary relation in equation 18 as fulfilled and $Z_{a}$ and $Z_{b}$ are as given in eq 16 .

The circuit shown in figure $6 \mathrm{~b}$, also will be shown to satisfy these conditions, and hence will represent an example with the desired transfer function. Although, as mentioned before, lattice networks cannot always be converted to equivalent bridged-tee networks, this particular circuit was chosen because it may be converted to an equivalent bridged-tee. As in the previous case, this lattice network is not generally convenient for use in phase shifting because for constant attenuation, simultaneous proportional adjustment of the resistance in two arms would be required. The equivalent bridged-tee has the advantage that the phase may be shifted by adjusting only one resistance element. It also offers the advantage of a common ground for one side of the input and output and one end of the adjustable phase-control resistor.

Examination of figure $6 \mathrm{~b}$, shows that, if we assume the $Q$ of the inductor to be high,

and

$$
\left.\begin{array}{l}
Z_{a} \doteq j \frac{X_{L} X_{C}}{2 X_{C}-X_{L}}, \\
Z_{b}=2 R_{2}-j X_{C} .
\end{array}\right\}
$$

These forms for $Z_{a}$ and $Z_{b}$ correspond to those given by eq 16 . If, in addition,

or

$$
\left.\begin{array}{l}
\frac{X_{L} X_{C}}{X_{L}-2 X_{C}}=2 X_{C}, \\
X_{L}=4 X_{C},
\end{array}\right\}
$$

the condition given in eq 18 is satisfied, and the circuit of figure 6b, provides an example of the desired phase shift network. From eq 16, 19, 20, and 21 , the transfer function may now be written

where

$$
\left.\begin{array}{rl}
\frac{E_{0}}{E_{i}} & =j \frac{R_{3}}{4 X_{C}} \epsilon^{+j 2 \theta}, \\
\theta & =\arctan \frac{X_{C}}{R_{3}+2 R_{2}}
\end{array}\right\}
$$

Equation 22, therefore, represents the transfer function for the circuit of figure $6, \mathrm{~b}$, provided

and

$$
\left.\begin{array}{l}
Q \text { (inductor) } \gg 1, \\
X_{L}=4 X_{C} \gg 2 R_{3} .
\end{array}\right\}
$$

Under these conditions, simultaneous variation of the resistances $R_{2}$ in the circuit of figure $6, \mathrm{~b}$, shifts the phase without varying the attenuation.

Figure 7 shows the equivalent bridged-tee network [4] corresponding to the lattice of figure $6, \mathrm{~b}$. The transfer function for this bridged-tee network is the same as that given in eq 22 for the corresponding lattice network of figure $6, \mathrm{~b}$. In this bridgedtee network (fig. 7), the phase may be shifted, without variation in attenuation, by varying the single resistance $R_{2}$. Examination of eq 22 shows that the magnitude of the attenuation is proportional to the ratio $R_{3} / 4 X_{C}$. This ratio must, however, be consistent with the relation given with eq 23 , in order 


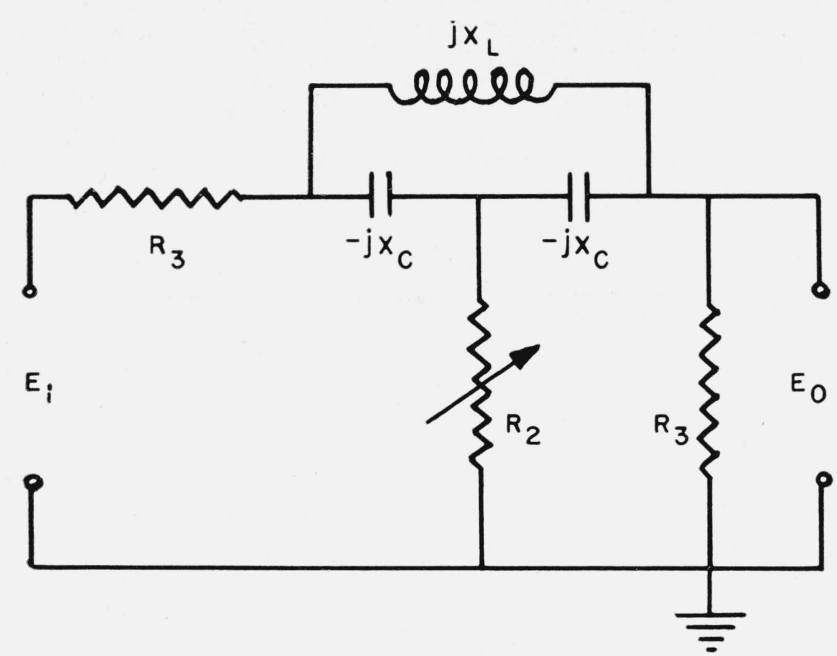

FIGURE 7. Bridged-tee equivalent to lattice network of figure $6, b$.

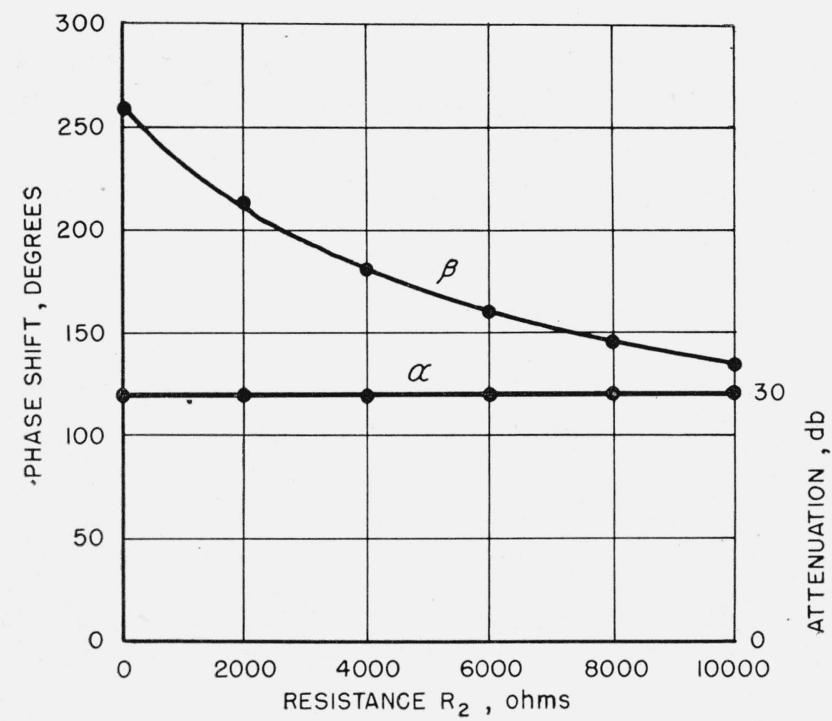

a.

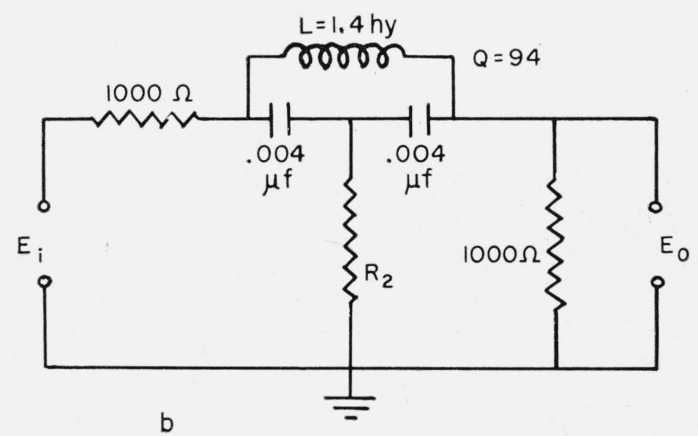

Figure 8. Attenuation with phase characteristics for bridged-tee network with variable resistance control $(f=4,170) \sim$.

that the attenuation remain constant with variatiou in phase when $R_{2}$ is varied.

Attenuation and phase characteristic curves, with phase shift and attenuation shown as functions of control resistance $R_{2}$, are given in figure 8 , a, for the bridged-tee network of figure $8, \mathrm{~b}$, operating at $4,170 \mathrm{c} / \mathrm{s}$.

\section{Geometrical Interpretation}

Equation 1 shows that the transfer function for the phase shift networks discussed in this paper may be considered as the difference between two admittances, except for a constant multiplier.

Figure 9, a, shows plots of the admittance loci $y_{a o}$ and $y_{b o}$ for the bridged-tee circuit of figure 3, wherein the phase is shifted by varying the reactance without producing any change in attenuation. Examination of eq 5 shows that, for this circuit, $y_{a o}$ is a constant vector independent of reactance $X$, whereas $y_{b o}$ is a vector whose origin rests at the extremity of the horizontal diameter of a circular locus and whose terminus moves along the circle as the reactance $X$ is varied. Examination of figure 9 , a, shows that if the length of the vector $y_{a_{0}}$ is made equal to the radius of the circular locus for $y_{b o}$, the difference $y_{a o}-y_{b o}$, representing the transfer function for the circuit of figure 3, will appear as a vector of constant length rotating in a circle, as pictured in figure $9, \mathrm{~b}$. The attenuation is thus seen to remain constant when the phase is changed by adjusting the value of the reactance $X$. These relations are represented analytically in eq 5 to 7 , inclusive. Equation 6 gives the analytical relation corresponding to the equality in length of the vector $y_{a_{o}}$ with the radius of the circular locus for $y_{b o}$, as pictured in figure 9 , $a$, and, $b$.

Figure 10, a, shows plots of the admittance loci $y_{a o}$ and $y_{b o}$ for the bridged-tee circuit of figure 7 , wherein the phase is shifted without variation in attenuation by changing the value of the resistance. Examination of eq 17 shows that for this circuit, $y_{a o}$ is a constant vector (along the axis of imaginaries) independent of resistance $R_{b}$, whereas $y_{b o}$ is a vector whose origin rests at the extremity of the vertical diameter of a circular locus and whose terminus moves along the circle as the resistance $R_{b}$, is varied. Examination of figure 10, a, shows that if the length of the vector $y_{a o}$ is made equal to the radius of the circular locus for $y_{b o}$, the difference $y_{a 0}-y_{b o}$, representing the transfer function for the circuit of figure
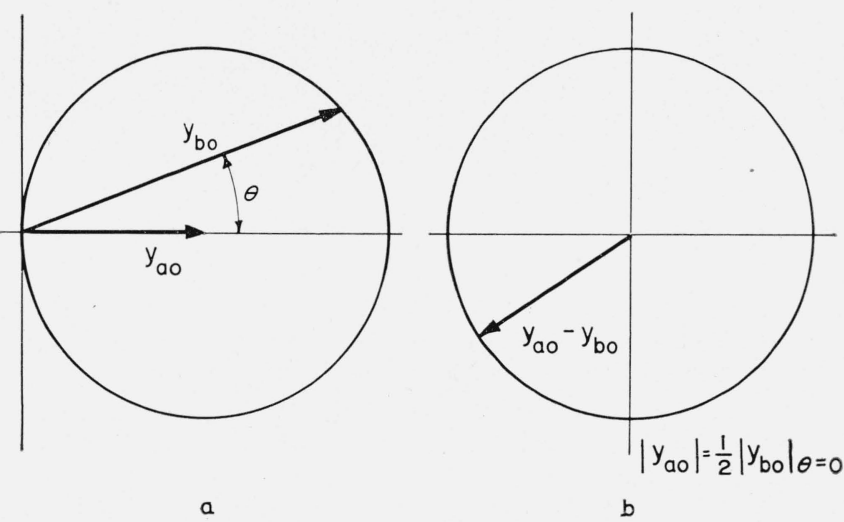

b

Figure 9. Admittance loci for bridged-tee of figure 3. 


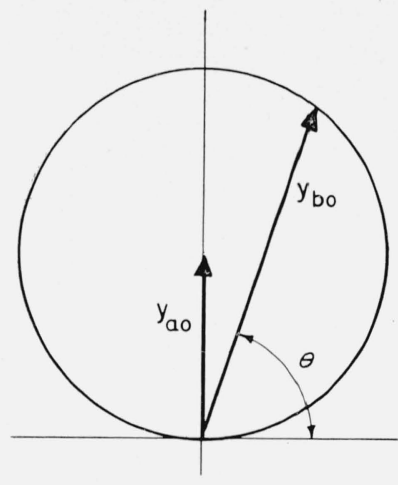

a

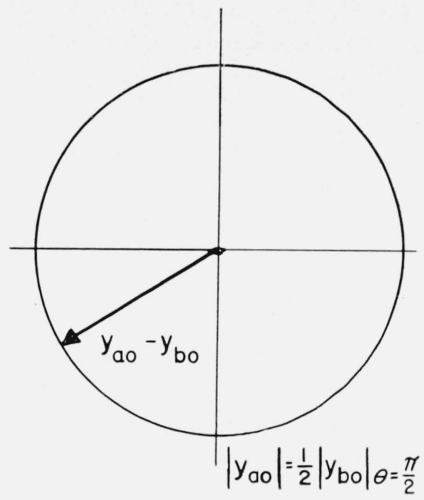

b
Figure 10. Admittance loci for bridged-tee of figure $\%$.

7, will appear as a vector of constant length rotating in a circle, as pictured in figure $10, \mathrm{~b}$. The attenuation is thus seen to remain constant for change in phase produced by changing the value of resistance $R_{b}$. These relations are represented analytically in eq 17 to 19 , inclusive. Equation 18 gives the analytical relation corresponding to the equality in length of the vector $y_{a o}$ with the radius of the circular locus for $y_{b o}$, as pictured in figure 10, a, and b.

\section{Related Null Network}

Referring to figure 9a, which shows plots of admittance loci related to the bridged-tee circuit of figure 3 , it can be seen that if $y_{a o}$ is made equal in magnitude to the diameter of the circular locus for $y_{b o}$, as shown by the dashed line of figure 9 , a, the difference $y_{a o}-y_{b o}$ will be a circular locus with the pole at the extremity of the horizontal diameter. Hence, as the reactance $X$ is varied continuously, the resultant vector will shrink to a null when the vectors for $y_{a o}$ and $y_{b o}$ become equal. The corresponding analytical conditions can be derived readily from the relations given in eq 5. This manipulation results in the well-known relations for this bridged-tee when used as a null network.

It is interesting to observe that the only adjustment required to change the null network to the phase shift network of figure 3 is to change $R_{1}$ from $R_{1}=4 R_{2}$, for the null condition, to the value given by eq 9 for the bridged-tee phase shift network.

\section{Impedance of Bridged-Tee Networks}

The image impedance of the symmetrical lattice of figure 1 (with $R_{3}$ omitted) is

$$
z_{o}=\sqrt{Z_{a} Z_{b}}
$$

For the lattice network of figure 2,b, and its equivalent bridged-tee shown in figure 3 (with $R_{3}$ omitted), the image impedance may be determined from the values of $Z_{a}$ and $Z_{b}$ given in eq 8 . If $2 X_{L}=X_{C 1}$

$$
z_{0}=\sqrt{R_{1} R_{2}}
$$

This is the input impedance the bridged-tee of figure 3 would have if $R_{3}$ in the input were shorted, and the load were given the value

$$
R_{3}=z_{o}=\sqrt{R_{1} R_{2}} .
$$

Solving eq 9 and 26 simultaneously, we obtain

$$
R_{i}=R_{3}=4 R_{2},
$$

for the input impedance of the bridged-tee of figure 3 if $R_{3}$ in the input were shorted. The impedance looking back into this network from the load is

$$
R_{0}=R_{3}=4 R_{2},
$$

if the source impedance is equal to $R_{3}$. The input and output impedances given by eq 27 and 28 for the bridged-tee of figure 3 are minimum values existing when $2 X_{L}=X_{C 1}$. These impedances become reactive when the phase is shifted by varying the circuit reactance from this value.

The input and output impedances of the bridgedtee of figure 7 may be shown to vary from $-j 4 X_{C} / 3$ when $R_{2}=0$ to $-j 4 X_{C}$ when $R_{2}=\infty$. For intermediate values of $R_{2}$, these impedances include a resistive component.

\section{Applications}

The bridged-tee phase shifting circuits described in this paper were developed originally for use with variable-resistance, or variable-reactance telemetering pickup devices to produce phase modulation in multiplex time-division telemetering systems and frequency modulation in multiplex frequency-division telemetering systems. Other possible applications include use as a phase shifter in servo loops including two-phase motors, and use as a phase modulator at high frequencies. The circuit of figure 5 , b, with phase and attenuation characteristics shown in figure 5 , a, was demonstrated successfully in the laboratory as a phase modulator at $29 \mathrm{Mc} / \mathrm{s}$. In this application, modulation was effected by variation of shunt capacitance with a reactance modulator. Alternately, the shunt capacitance could be varied by utilizing a barium-strontium titanate element whose capacitance changes with variation in modulating voltage.

For voltage modulation of phase, the bridged-tee of figure 7 was tested with the variable resistance $R_{2}$ replaced by a biased varistor as shown in figure $11, \mathrm{~b}$. Static tests at 7,250 kc indicated excellent linearity of phase versus bias voltage over a $90^{\circ}$ phase shift, as shown in the experimental curve of figure 11, a. The nonlinear characteristic of the biased varistor compensates for the nonlinear phase characteristic of the bridged-tee network, resulting in a remarkably linear phase response over the $90^{\circ}$ range. As seen from the theory of the bridged-tee phase shifter above, this range of modulation is effected with no change in attenuation. The loading effect of the net- 


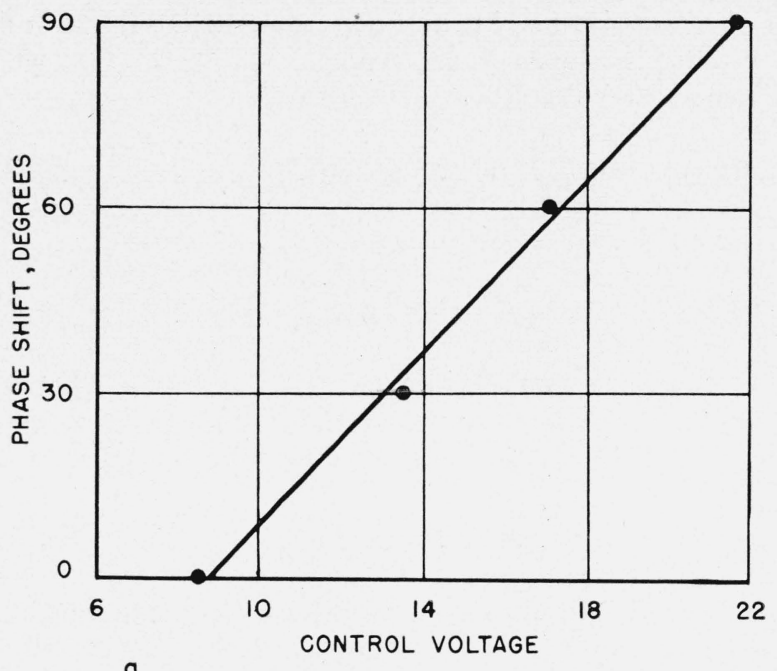

a

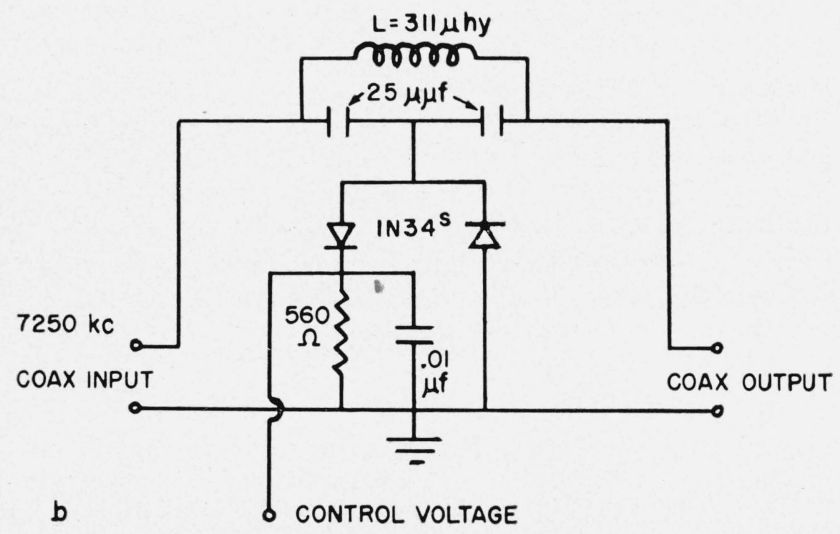

Figure 11. Phase shift versus control voltage for bridged-tee phase modulator $(f=7,250 \mathrm{kc})$. work during modulation is not generally serious, since the input reactance of the network decreases to only $-j 4 X_{C} / 3$ ohms when $R_{2}$ becomes zero.

Another application of the bridged-tee of figure 3 is suggested by the previously mentioned property of this network of giving constant attenuation with variation in frequency as shown in figure $4, \mathrm{~b}$. Two bridged-tee networks in tandem, properly adjusted, result in a constant phase shift independent of frequency over a wide range. This combination of bridged-tee networks may have advantages over lattice networks that have been described recently $[5,6]$ for this purpose, particularly since the bridged-tee is single-ended and functions when loaded. Hence, no phase-inverting and isolating vacuum tube amplifiers should be required with this bridged-tee arrangement, although the attenuation through the two bridged-tee networks may be a disadvantage in some applications.

\section{References}

[1] F. E. Terman, Radio engineers' handbook, p. 918 (McGraw-Hill Book Co., New York, N. Y., 1943).

[2] I. A. Greenwood, Jr., J. V. Holdam, Jr., and D. MacRae, Jr., Electronic instruments, Radiation Laboratory Series 21, 230 (McGraw-Hill Book Co., Inc., New York, N. Y., 1948).

[3] E. A. Guillemin, A method of representing the insertion loss and angle of symmetrical networks (manuscript circulated to staff members of Radiation Laboratory during World War II).

[4] H. Lauer, R. Lesnick, and L. Matson, Servomechanism fundamentals, 1st ed., p. 158 (McGraw-Hill Book Co., Inc., New York, N. Y., 1947).

[5] R. B. Dome, Wide-band phase shift networks, Electronics 19, 112 to 115 (Dec. 1946).

[6] David G. C. Luck, Properties of some wide-band phaselifting networks, Proc. IRE 37, 147 to 151 (Feb. 1949).

Washington, March 28, 1950.

\title{
Stability of Dextrose Solutions of Varying $\mathrm{pH}$
}

\section{By Emma J. McDonald}

\begin{abstract}
A study has been made of the rate of the initial decomposition reaction taking place in dextrose solutions of varying $\mathrm{pH}$. From the change in direct optical rotation over a period of time velocity constants have been calculated for eight values of $\mathrm{pH}$. The results indicate that dextrose solutions are most stable at about $\mathrm{pH} 4$.
\end{abstract}

\section{Introduction}

A great deal of work has been reported in the literature concerning the decomposition of reducing sugars in acid and in alkaline solutions. However, there is little to be found that gives an explanation or measure of the relation that exists between the $\mathrm{pH}$ and the stability of aqueous dextrose solutions. The wide use of dextrose solutions as standards in methods of sugar analysis has led to this investigation. In addition, aqueous solutions of dextrose find application in the practice of medicine where the solutions are stored in a sterile condition in ampoules or in bottles. A great variety of food products, both naturally occurring and manufactured, contain dextrose, often as one of the main constituents. Fruit juices, honey, table sirups, and molasses are examples of this group.

In the presence of aqueous acids, dextrose undergoes condensation reactions, forming disaccharides and possibly higher oligosaccharides. The products of this primary reaction, as is to be expected, are of great variety as the mode of attachment of two dextrose molecules is unselective and depends upon the 